\title{
Intervención en Atención Temprana en un caso de Potocki-Lupski
}

\section{Early Care Intervention in a Case of Potocki-Lupski Syndrome}

\author{
Zaira Morales ${ }^{1}$ y Gloria Martin-Lepe ${ }^{2}$ \\ ${ }^{1}$ Universidad de Huelva, España ${ }^{2}$ Hospital de San Juan de Dios de Sevilla, España
}

\begin{abstract}
Resumen: El síndrome de Potocki-Lupski es un síndrome genético causado por una microduplicación en el cromosoma 17p11.2. La mayoría de los casos aparecen de novo y se caracterizan por una sintomatología heterogénea que suele incluir hipotonía, retraso en el desarrollo, anomalías congénitas y alteraciones en el comportamiento de diversa índole que en algunos casos pueden estar asociados a trastornos del espectro autista. Los trabajos sobre intervención y tratamiento psicológicos sobre este síndrome son escasos. En el presente trabajo se describe la evaluación e intervención desde la Atención Temprana llevada a cabo con un niño de dos años diagnosticado con dicho síndrome durante 18 meses. Los resultados muestran una evolución positiva a nivel clínico en las distintas áreas del desarrollo, añadiendo una perspectiva práctica a los estudios de menores que padecen este síndrome bastante desconocido, además de apoyar una sintomatología común a la hallada en otros estudios.

Palabras clave: atención temprana, estudio de caso único, Síndrome de Potocki-Lupski, intervención, síndromes raros
\end{abstract}

\begin{abstract}
Potocki-Lupski syndrome is a genetic syndrome caused by a microduplication on chromosome 17p11.2. Most cases appear de novo and are characterized by a heterogeneous symptomatology that usually includes hypotonia, developmental delay, congenital anomalies and alterations in behavior of various kinds that, in some cases, may be associated with autism spectrum disorders. Papers on psychological intervention and treatment of this syndrome are scarce. This paper describes the evaluation and intervention by Early Care carried out over a period of 18 months for a two-year-old child diagnosed with this syndrome. The results show a positive evolution at a clinical level in the different areas of development,
\end{abstract}

\footnotetext{
Zaira Morales (iD https://orcid.org/0000-0002-4907-7389 es profesora asociada del Departamento de Psicología Clínica y experimental de la Facultad de Educación, Psicología y Ciencias del Deporte de la Universidad de Huelva.

Gloria Martin-Lepe es Psicóloga sanitaria en el Centro de atención temprana del Hospital de San Juan de Dios de Sevilla.
}

La correspondencia de este artículo debe ser enviada a Zaira Morales al email: zaira.morales@dpsi.uhu.es

(cc) BY-NC-ND Este es un artículo Open Access bajo la licencia CC BY-NC-ND. 
adding a practical perspective to studies of minors with this rather unknown syndrome, as well as supporting a symptomatology similar to that found in previous studies.

Keywords: early Care, single-case study, Potocki-Lupski Syndrome, intervention, rare syndromes

El síndrome de Potocki-Lupski (PTLS) es una enfermedad genética rara causada por una duplicación en el cromosoma 17 (17p11.2). El tamaño de la duplicación suele ser reducido (3.7 Mb aproximadamente) y el origen, en la mayor parte de las ocasiones, es de novo (Potocki et al. 2007, Zhang et al. 2010). Suele señalarse como principal responsable de la aparición del PTLS al gen RAI1 (del inglés Retinoic Acid Induced 1) (Lee et al., 2012), aunque se han identificado diversos genes que también pueden afectar (Potocki et al.2007, Yuan et al., 2015).

El PTLS es un síndrome relativamente novedoso. Aunque a finales de la década de los 90 y principios del año 2000 ya se detectaron casos con la mencionada duplicación (Brown et al., 1995; Potocki, et al., 2000), no es hasta el año 2007 cuando en un nuevo estudio se profundiza en las características del síndrome, acuñando el nombre de la investigadora e investigador que dirigían el proyecto (Potocki et al, 2007). A partir de entonces, teniendo en cuenta a la población infantil, los trabajos que han ido apareciendo en la literatura científica lo han hecho principalmente desde la perspectiva genética (Potocki et al., 2007; Yuan et al., 2015), en la mayor parte son estudios de caso único (Bravo et al., 2013; Domenico et al., 2018; Fernández et al., 2016; McCool et al., 2017) y describen sobre todo aspectos clínicos.

Los estudios llevados a cabo relacionados con la clínica del síndrome apuntan a unas características heterogéneas en cuanto a la sintomatología, presentándose, aunque no en todo los casos ni con la misma intensidad, rasgos faciales dismórficos (Lee et al., 2013; Martin et al., 2008; McCool et al., 2017; Potocki et al., 2007), retraso en el desarrollo (Lee et al., 2013; Potocki et al., 2007; Soler-Alfonso et al., 2010), hipotonía (Martin et al., 2008; Potocki et al., 2007; Soler-Alfonso et al., 2010), discapacidad intelectual (Martin et al., 2008; Potocki et al., 2007; Soler-Alfonso et al., 2010; Treadwell-Deering et al., 2010) alteraciones en el sueño (Potocki et al., 2007), dificultades en la alimentación (Martin et al., 2008; Potocki et al., 2007; Soler-Alfonso et al., 2010; Yuan et al., 2015), problemas en el lenguaje (Lee et al., 2013; Lee et al., 2012; Martin et al., 2008; McCool et al., 2017; Potocki et al., 2007; Yuan et al., 2015), anomalías congénitas (Bravo et al., 2013; Martin et al., 2008; Potocki et al., 2007; Yuan et al., 2015) y alteraciones en el comportamiento y sociales, llegando en algunos casos a presentar sintomatología relacionada con trastornos del espectro autista (Martin et al., 2008; McCool et al., 2017; Potocki et al., 2007; Treadwell-Deering et al., 2010; Yuan et al., 2015). En la Tabla 1 se resumen los signos y síntomas comunes en el síndrome de Potocki-Lupski, así como la frecuencia con la que suelen observarse (Domenico et al., 2018; GARD, 2018; Potocki et al. 2007; Treadwell-Deering et al., 2010).

En relación a la prevalencia del síndrome no se han hallado datos concretos, ni a nivel general ni a nivel de población española en particular. Se le considera una enfermedad rara ya que su incidencia se estima en 1 caso por cada 20.000-25.000 nacidos (Bissell et al., 2018; Treadwell-Deering et al., 2010) pero también se apunta a la posibilidad de que exista un infradiagnóstico (Potocki et al., 2000). La asociación de padres y madres de niños diagnosticados en España con el síndrome Potocki-Lupski nos informa que en octubre de 2018 son veintinueve familias las que habían establecido contacto con ellos, concretamente padres de 13 chicos y 16 chicas.

A nivel de intervención, los trabajos encontrados son escasos y refieren tratamientos según las características particulares del caso (McCool et al., 2017; Soler-Alfonso et al., 2010; Fernández et al., 2016) posiblemente debido, por un lado, a la baja frecuencia de publicaciones que hay de este síndrome en la infancia, y por otro a la heterogeneidad de la sintomatología.

Tratándose de la atención a infantes, la Atención Temprana (AT) muestra ser una estrategia eficaz en la prevención y compensación de los efectos causados por los déficits ya sean por cuestiones evolutivas, biológicas o sociales (para más información véase Robles-Bello y Sánchez-Teruel, 2013). Y aunque no se han encontrado estudios en casos de Potocki-Lupski tratados con AT, ésta se muestra necesaria (Sánchez-Teruel, 2011), debido a que ha quedado patente su utilidad a niveles prácticos, tanto a nivel de evaluación como de intervención, 
Tabla 1. Sintomatología y frecuencia de presentación en el síndrome de Potocki-Lupski

\section{\% de presentación Signos y síntomas}

$80-99 \%$

(muy frecuente)

\section{Hipotonía.}

Retraso en el desarrollo.

Sintomatología asociada a los trastornos del espectro autista (TEA).

Trastorno por déficit de atención e hiperatividad.

Dificultades en el área del lenguaje: retraso en el lenguaje expresivo, disartria, disfasia, ecolalia.

Discapacidad intelectual (media-moderada).

Problemas con la alimentación durante los primeros años de vida.

Apnea del sueño.

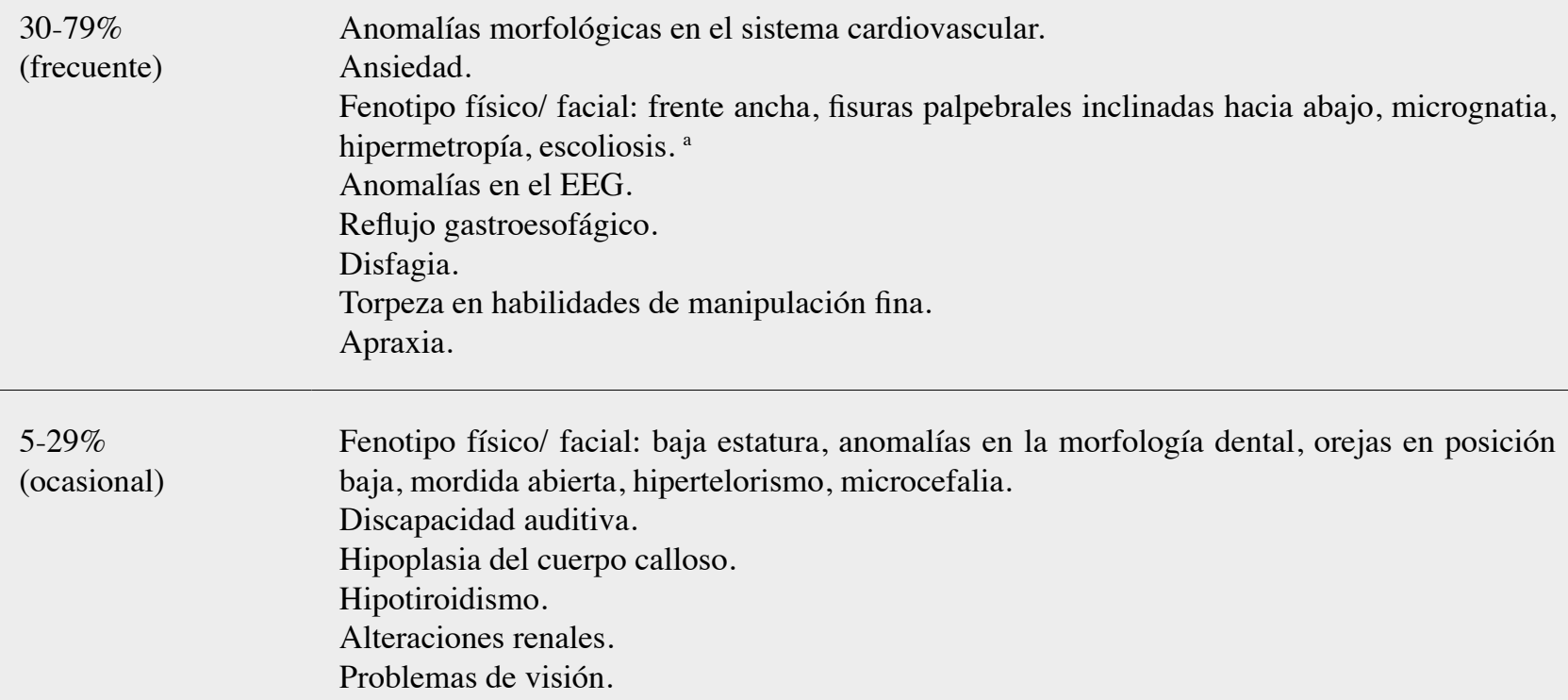

$30-79 \%$

(frecuente)

Anomalías morfológicas en el sistema cardiovascular.

Ansiedad.

Fenotipo físico/ facial: frente ancha, fisuras palpebrales inclinadas hacia abajo, micrognatia, hipermetropía, escoliosis. ${ }^{\text {a }}$

Anomalías en el EEG.

Reflujo gastroesofágico.

Disfagia.

Torpeza en habilidades de manipulación fina.

Apraxia.

$5-29 \%$

(ocasional)

Fenotipo físico/ facial: baja estatura, anomalías en la morfología dental, orejas en posición baja, mordida abierta, hipertelorismo, microcefalia.

Discapacidad auditiva.

Hipoplasia del cuerpo calloso.

Hipotiroidismo.

Alteraciones renales.

Problemas de visión.

${ }^{a}$ Los rasgos faciales en la mayoría de los pacientes no son estrictamente dismórficos. Sin embargo, presentan muchas características similares.

Fuentes: Domenico et al., 2018; GARD, 2018; Treadwell-Deering et al. 2010

en casos de trastornos genéticos en general, y de enfermedades raras en particular (Robles-Bello, 2016; Robles-Bello et al., 2018; 2014; 2017).

Teniendo en cuenta todo lo anterior, que es necesario hacer visible tratamientos eficientes y efectivos en este síndrome, que cuanto más temprano se inicie la intervención menos secuelas presentará el menor en el futuro, y que la AT ha mostrado su utilidad en la evaluación y tratamientos de menores con trastorno genéticos, en este trabajo el objetivo es presentar un caso de intervención en Atención Temprana en un menor diagnosticado de PTLS y sus efectos.

\section{Método}

\section{Diseño}

Estudio de caso único que recoge evaluación diagnóstica e intervención terapéutica. La evaluación tomó un carácter longitudinal, con tres mediciones, a los 23, 28 y 41 meses de edad del menor. Entre las evaluaciones se aplicó un programa de estimulación global con objetivos para las distintas áreas del desarrollo. 


\section{Participante}

El paciente es un menor varón que acude a la unidad de atención temprana del hospital San Juan de Dios de Córdoba desde los 10 meses, diagnosticado de retraso madurativo, atendiendo al protocolo de seguimiento a niños recién nacidos de riesgo (Pons-Tubío, 2009). En ese momento tenía adquirido el control cefálico e iniciaba la sedestación. No volteaba de decúbito supino a decúbito prono. Se desplazaba sobre la barriga, muy despacio. Presentaba hipotonía axial. Cogía objetos que se le mostraban llevando las dos manos al objeto.

Como información perinatal decir que la madre se quedó embarazada a través de fecundación in vitro cuando contaba con 38 años de edad. Es el primer y único embarazo hasta ese momento. El transcurso del mismo fue normal, con finalización a las 34 semanas de gestación por parto natural con rotura previa de la bolsa.

En el momento del nacimiento el niño presentó reflujo vesículo-ventral y aumento del diámetro del riñón izquierdo. Su peso fue de 1,840 gramos y midió $44 \mathrm{~cm}$ al nacer, permaneciendo hospitalizado durante 28 días en la unidad de neonatología. Durante los tres primeros meses de vida se alimentó por lactancia mixta.

A los 12 meses de edad cronológica (10.5 meses de edad corregida), se desplazaba gateando, realizaba cambios posturales y se ponía en pie con ayuda. Iniciaba emisiones vocálicas aisladas. Se observaba sonrisa social. Prestaba escasa atención y no respondía al nombre.

A los 18 meses fue diagnosticado con el síndrome de Potocki-Lupski mediante la técnica de amplificación de sondas dependientes de ligandos múltiples (MLPA).

A los 23 meses de edad cronológica (21.5 meses de edad corregida) se inició la intervención que aquí se describe durante un periodo de 18 meses. Antes de iniciar el programa, el niño recibía una sesión de estimulación cognitiva a la semana, dos sesiones de estimulación de fisioterapia, una sesión de natación especializada y una sesión de psicoeducación en la escuela infantil a la que asistía. Además, se encontraba en seguimiento en los siguientes servicios médicos: neuropediatría, nefrología, oftalmología, digestivo, rehabilitación, neonatología y unidad de salud mental infanto-juvenil.

\section{Instrumentos de evaluación}

Para realizar una evaluación adecuada a un menor, la valoración debe tomar un carácter interdisciplinar y se debe atender a las distintas áreas del desarrollo (cognición, lenguaje, motricidad, comportamiento, funcionamiento social y emocional y conducta adaptativa), además de tener en cuenta otros aspectos influyentes en el desarrollo y adaptación del menor, como los recursos familiares y del entorno (GAT, 2011). Los principales objetivos de la valoración son dos, detectar si el menor tiene un desarrollo acorde a unos patrones de normalización, así como analizar y describir sus capacidades y principales dificultades, para poder generar pautas de intervención eficaces (Arnedo, Montes, Bembibre y Triviño, 2015; Costas, 2009).

Con el objetivo de elaborar un programa de tratamiento adecuado se siguió para la evaluación las indicaciones de evaluación multicomponente propuesta en el Libro Blanco de Atención Temprana (GAT, 2011), para ello, se usó como técnicas la observación directa, la entrevista de tipo semiestructurada y abierta a padres y educadores y la aplicación de pruebas en situación estructurada.

La valoración se inició con una entrevista a la familia y observación clínica del menor a través del juego. Posteriormente, se pasó a la selección de dos escalas de desarrollo, comúnmente utilizadas en el ámbito de evaluación de la atención temprana (Candel, 2003; Costas, 2009; Robles-Bello y Sánchez-Teruel, 2006): la Escala de desarrollo psicomotor de la primera infancia Brunet-Lézine Revisada (BLR-E; Josse, 1997) y el Inventario de desarrollo Battelle (BDI; Newborg et al., 1988), único aplicado en la tercera evaluación; y dos pruebas muy usadas en el ámbito de la atención temprana como guía para la propuesta de objetivos de trabajo (Robles-Bello y Sánchez-Teruel, 2006), aplicadas en la primera evaluación e iniciación de la intervención a los 23 meses así como a los 28 meses de edad cronológica, la Guía Portage (Bluma y Reinaud, 1978) y la Rueda del desarrollo (Cabrera et al., 2002).

En las entrevistas iniciales se tomó información sobre los antecedentes familiares, desarrollo pre/peri/post-natal, anamnesis, tratamientos e intervenciones previas... se utilizó principalmente como técnica la escucha activa. 
Entre otros datos los padres cuentan que el pequeño era un niño "flojo" para mamar y poco "comilón", no presentaba problemas de sueño y lo describieron como un bebé tranquilo, que se movía poco en la cuna y flojo a nivel de extremidades. No describieron antecedentes familiares de relevancia.

El niño no mostró en ningún momento rechazo hacia la terapeuta, ni hacia el entorno donde tuvo lugar la valoración. Mantenía una actitud positiva hacia su madre, se mostraba cariñoso con ésta, aunque solía ser ella quien iniciaba la interacción. Antes de iniciar la evaluación, se hizo uso de algunas sesiones para el establecimiento de un clima de confianza. Durante estas sesiones se realizó una aproximación gradual al niño y se anotó sus preferencias, así como los estímulos que le resultaban aversivos.

Con la observación directa se observó sonrisa frecuente, sin causa que la generase, así como preferencia por juegos sensoriales (estímulos sonoros, luminosos).

Se percibió en el menor rasgos fenotípicos característicos del síndrome de Potocki-Lupski como frente ancha, fisuras palpebrales inclinadas hacia abajo, micrognatia, anomalías en la morfología dental y orejas en posición baja, además de baja estatura. Además, coincidiendo con otros casos descritos en la literatura sobre este síndrome, también mostraba hipotonía, torpeza motora, dificultades en el sueño y retraso en el desarrollo y en el lenguaje expresivo. Asociado al trastorno por déficit de atención e hiperactividad (TDAH) se identificó alteración en la atención mantenida y selectiva y gran inquietud motora (deambulación, dificultad para mantenerse sentado y respetar turnos en juegos circulares). Entre las características propias del trastorno de espectro autista (TEA) se observó la presencia de alteraciones en dos niveles: déficits persistentes en comunicación e interacción social y patrones repetitivos de conducta (escasa intención comunicativa, contacto ocular casi inexistente, no respondía a su nombre, ni seguía órdenes sencillas). Iniciaba señalando con función protodeclarativa. A nivel de lenguaje expresivo se observó emisiones de sonidos guturales, balbuceo y sonidos vocálicos. No mostraba juego simbólico ni interés por los iguales. Era cariñoso con adultos conocidos, aunque con escasa iniciativa en la interacción. Presentaba aleteo ante la excitación, rabietas frecuentes y respuestas de ansiedad ante estímulos que implicasen inestabilidad.

La aplicación de las pruebas estandarizadas se llevó a cabo en presencia de la madre, ya que la colaboración del menor era mejor en esta situación. Las que se le aplicó al inicio fueron las que a continuación se describen:

Los ítems de la etapa de 0-24 meses de la Guía Portage (Bluma y Reinaud, 1978). Esta prueba de evaluación permite determinar las capacidades generales de los niños desde el nacimiento hasta los seis años. Se compone de 578 fichas que valoran cinco áreas de desarrollo: socialización, lenguaje, autoayuda, cognición y desarrollo motriz. Cada ficha indica el área de desarrollo que evalúa, la edad de aplicación, el objetivo a conseguir y la descripción detallada de las actividades a realizar para conseguir dicho objetivo dentro del proceso de intervención. Además, incluye un bloque con orientaciones para la estimulación del niño, que pretende servir de guía a la familia y/o educadores en la propuesta de situaciones de aprendizaje. Se trata de actividades dirigidas a niños de 0 a 4 meses (meses cronológicos o madurativos).

La Escala de observación sistemática de 0 a 48 meses, elaborada por Radio Ecca (Cabrera et al., 2002). Esta escala se incluye dentro del protocolo de pruebas que se usan en esta entidad. Recoge de forma gráfica la valoración de las habilidades del desarrollo, atendiendo a cinco áreas: área motora fina y gruesa, área perceptivo-cognitiva, área de lenguaje y área social.

La Escala de desarrollo psicomotor de la primera infancia Brunet-Lézine Revisada (BLR-E; Josse, 1997) se trata de una prueba de 150 ítems que se responde con 1 ó 0 según si la habilidad que se evalúa está presente o no. Valora el desarrollo de los menores hasta los 2 años y medio y permite realizar una estimación de la edad, perfil de desarrollo y cociente intelectual. Evalúa cuatro áreas: postural, coordinación, sociabilidad y lenguaje. El resultado de sumar los diferentes ítems proporciona la edad de desarrollo (ED). El resultado de dividir la edad de desarrollo por la edad real o cronológica (EC) refleja el cociente de desarrollo (CD). Estos resultados se obtienen para cada área (cociente de desarrollo parcial) y para el conjunto total de ítems (cociente de desarrollo global) (Costas, 2009).

El Inventario de desarrollo Battelle (BDI; Newborg et al., 1988), evalúa las habilidades fundamentales del desarrollo del niño desde el nacimiento hasta los 7 años y 11 meses. Consta de una batería completa y un test de cribado. La batería está formada por 341 ítems, existiendo una versión reducida con 96 ítems. Se puntúa de $0-2,2$ puntos si la respuesta se ajusta al criterio establecido, 1 punto si el niño intenta resolver la prueba, pero 
no lo consigue, 0 en el caso de respuesta nula o muy deficitaria. A partir de las puntuaciones directas se pueden obtener datos de percentiles, cocientes de desarrollo, edades equivalentes y puntuaciones z y T, para cada área y a nivel global (Costas, 2009; Newborg et al. 1988). Valora cinco áreas: personal/social, adaptativa, motora, comunicación y cognición. Éstas a su vez están divididas en subáreas.

Como resultado de la evaluación inicial (las puntuaciones se detallan en el apartado resultados) se llega a la conclusión de que el menor cumplía criterios para recibir tratamiento de estimulación en atención temprana ya que se observó una desviación de la normalización en su desarrollo (GAT, 2011). Por tanto, se hacía necesario el desarrollo de un programa de estimulación específico.

Posteriormente se vuelve a evaluar al niño para ver el avance según el programa específico. Estas evaluaciones, como se indicó anteriormente, se llevaron a cabo a los 28 y 41 meses

\section{Consideraciones éticas}

El trabajo realizado respeta los principios incluidos en la Declaración de Helsinki (Mazzanati, 2011). Previamente, se informó a los padres de manera verbal sobre el estudio y autorizaron dicha participación voluntaria por escrito. Ninguna de las tareas realizadas generó molestias en el menor o su familia.

\section{Intervención}

Se desarrolló un programa de estimulación específico tras detectar las necesidades del niño en las distintas áreas del desarrollo, la situación y posibilidades de su familia y entorno, siguiendo la recomendación del Libro blanco de atención temprana (GAT, 2011), haciendo especial énfasis en las áreas socio-comunicativas, ya que fue donde se detectó mayores dificultades.

El programa se implementó en un primer momento con una temporalización de cuatro meses, donde se hizo la siguiente evaluación, para posteriormente ampliarlo trece meses más.

Se utilizó como técnicas para la intervención principalmente las derivadas de los principios de modificación de conducta, destacando entre otras el papel del refuerzo positivo (aplicado inicialmente a razón fija) y la extinción de las conductas inadecuadas (como rabietas o llamadas de atención). También se usó constantemente técnicas basadas en el juego en función de su edad (para más información véase Fuentes-Biggi et al., 2006). Como estrategias generales de intervención se trabajó con apoyos visuales, la utilización de un lenguaje adaptado y la exposición a situaciones de aprendizaje sin error (sistema de ayuda). Además, se planificó un entorno estructurado y se descompusieron las tareas en pasos (encadenamiento), se le propuso a la familia usar el sistema comunicativo de intercambio de imágenes (PECS), sin embargo, esta optó por no utilizarlo en principio. Se utilizó el acompañamiento del lenguaje oral con signos, siguiendo como referencia el programa de comunicación total de Benson Schaeffer (Schaeffer et al.,1980). Inicialmente, se usó signos para dar apoyos a estructuras orales frecuentes en la interacción con el menor ("dame", "más", "se acabó") o de gran interés para el niño ("pompas", "agua"). La estrategia de segmentación fonológica se utilizó en la producción de los signos. Ésta consiste en reproducir el signo tantas veces como sílabas tenga la palabra verbal.

Se incide en la necesidad de seguir las rutinas de tratamiento, con períodos de trabajo en mesa y finalización de las tareas o juegos iniciados.

Durante la aplicación del programa, se acudió a tres tipos de actividades: actividades rutinarias (que ocurren regularmente), actividades planificadas (no suelen ocurrir normalmente si no son diseñadas) y actividades iniciadas por el niño (Candel, 2003; 2005). Se realizaron sesiones de 45 minutos una vez a la semana, tiempo que equivale a 1 UMAT, unidad de medida en atención temprana (GAT, 2011). Las sesiones solían seguir siempre la misma secuencia: inicio con tareas de baja exigencia para el menor, seguida de dos actividades que requerían mayor atención y esfuerzo, para terminar con alguna tarea de índole motora o de interés (ésta última solía desarrollarse fuera de la mesa). 
Durante las sesiones la madre estaba presente, lo que permitía que participara en las actividades y que se le pudiera ofrecer orientación, información y psicoeducación de manera conjunta, además de fomentar el trabajo conjunto y la generalización de los objetivos al entorno natural del niño.

Se propone en las distintas áreas de desarrollo los siguientes objetivos:

A nivel cognitivo: mejorar la atención y la exploración (trabajo en mesa, imitación-contraimitación, la respuesta al nombre, el aprendizaje de relación causa-efecto), aumentar la discriminación perceptiva (encajables, torres) y la adquisición de conceptos (esquema corporal, asociación de iguales, vocabulario básico).

A nivel social: mejorar el contacto ocular, la atención y juego conjunto (pompas, juegos circulares), fomentar el juego simbólico y la imitación. Además, se facilita a la familia pautas para trabajar en el entorno natural del niño.

En el nivel de autonomía personal: el agarre del biberón y la colaboración en el vestido-desvestido. En el área de la alimentación se propuso la introducción de semi-sólidos y de nuevos sabores.

En el área de comunicación-lenguaje: aumentar la intención comunicativa (señalado, uso de gestos), estimular el lenguaje oral (imitación de sonidos y onomatopeyas) y mejorar la comprensión del lenguaje (identificar objetos por su nombre, seguimiento de instrucciones sencillas).

A nivel motor: mejorar las habilidades de manipulación fina (garabateo, pinchos, enhebrado, plastilina), aumentar la coordinación y equilibrio (andar sobre distintas superficies, subir-bajar escaleras, juego con pelota) e iniciar el uso de estímulos motores que generaban ansiedad (corre pasillos, pelotas grandes, toboganes).

También se le facilitó a la familia algunas consideraciones a tener en cuenta en el hogar. Siguiendo a Candel (2003; 2005), se les informó sobre la importancia de despertar en el niño interés por el medio que le rodea, hablar al niño mirándole a la cara, ofrecer estímulos atractivos y propios de su edad, permitir que los observe y manipule, y exponerlo a estímulos habituales de su entorno son algunas pautas para esta primera consideración. Se les instruyó en la conveniencia de aprovechar las rutinas diarias para estimular al niño, seleccionar los momentos de alerta y receptividad para esta estimulación, respetando los tiempos del niño, sin sobrecargarlo. Se les resaltó la importancia de establecer una adecuada interacción

padres-hijo, buscando reforzar las respuestas socio-comunicativas del niño. Y se señaló la importancia de involucrar al niño en actividades funcionales.

En relación al medio, se destacó la necesidad de adaptar el entorno al menor, para favorecer su respuesta a los estímulos que se presentasen.

Tras la segunda evaluación, se reforzó los objetivos no conseguidos y se propuso los siguientes: iniciar el salto, aumentar su vocabulario y conceptos propios a su edad, realizar asociaciones básicas entre elementos presentados, conocer su nombre, utilizar palabras como dame o más para demandar de forma verbal, aumentar el tiempo de trabajo en mesa y el seguimiento de normas, y la disminución de conductas inadecuadas (llamadas de atención, conductas repetitivas).

Con la tercera evaluación se pretendió valorar el nivel de desarrollo conseguido, así como las necesidades presentes por áreas de desarrollo. Fue necesario proponer nuevos objetivos, además de fomentar la generalización de los aprendizajes adquiridos hasta el momento. Entre las nuevas propuestas se encontraban: iniciar el uso de triciclos, discriminar y separar elementos por categorías semánticas, responder a preguntas sencillas (qué, quién) y fomentar el juego con iguales.

\section{Resultados}

Los resultados medios de la Guía Portage (Bluma y Reinaud, 1978) obtenidos por el niño en la primera evaluación indicaban una edad de desarrollo de 6-12 meses en el área de socialización, 6-12 meses en el área de autoayuda, 3-6 meses en el área de lenguaje, 9-12 meses en el área cognitiva; y 12-18 meses en el área de desarrollo psicomotriz. Después de los primeros cuatro meses de intervención los resultados obtenidos fueron: 12-18 meses en el área de socialización; 6-12 meses en el área de autoayuda; 12 meses en el área de lenguaje; 18-24 meses en el área cognitiva; y 18-24 meses en el área de desarrollo psicomotriz. 
Los obtenidos según la valoración de los ítems superados por Escala de observación sistemática de 0 a 48 meses (Cabrera et al., 2002) , señalaban a una edad de 9-12 meses en el área motora fina, 16-18 meses en el área motora gruesa, 9-16 meses en el área perceptivo-cognitiva, 0-6 meses en el área del lenguaje y 0-6 meses en el área social. Después de los primeros cuatro meses de intervención señalan una edad madurativa de 16-18 meses en el área motora fina, 18-24 meses en el área motora gruesa, 16 meses en el área perceptivo-cognitiva, 6-12 meses en el área del lenguaje y 6-12 meses en el área social

Los resultados obtenidos con el Inventario de desarrollo Battelle se exponen en la Tabla 2. Las puntuaciones obtenidas situaron al menor en el percentil (PC) 1, con una desviación típica de -2.33 puntos. En la tercera evaluación, la puntuación de la subárea cognitiva puntúa en el PC 2 y una desviación típica de -2.05 puntos. La Figura 1 recoge las edades equivalentes obtenidas con esta prueba.

Tabla 2. Resultados obtenidos en el Inventario de desarrollo Battelle en las tres evaluaciones realizadas (23 meses, 28 meses, 41 meses)

\begin{tabular}{|c|c|c|c|c|}
\hline & & $1 E$ & $2 E$ & $3 E$ \\
\hline \multirow[t]{2}{*}{ P-S } & PD & 22 & 32 & 57 \\
\hline & $\mathrm{EE}$ & 5 & 9 & 18 \\
\hline \multirow[t]{2}{*}{ A } & PD & 19 & 22 & 28 \\
\hline & $\mathrm{EE}$ & 7 & 8 & 11 \\
\hline \multirow[t]{2}{*}{ M } & PD & 54 & 67 & 82 \\
\hline & $\mathrm{EE}$ & 13 & 17 & 26 \\
\hline \multirow[t]{2}{*}{ Com } & PD & 12 & 21 & 32 \\
\hline & $\mathrm{EE}$ & 5 & 11 & 18 \\
\hline \multirow[t]{2}{*}{$\operatorname{Cog}$} & PD & 18 & 24 & 32 \\
\hline & EE & 10 & $16-18$ & 26 \\
\hline \multirow[t]{2}{*}{$\mathrm{T}$} & PD & 125 & 166 & 231 \\
\hline & EE & 9 & 13 & 20 \\
\hline
\end{tabular}

Nota: $\mathrm{P}-\mathrm{S}=$ área personal-social; $\mathrm{A}$ = área adaptativa; $\mathrm{M}$ = área motora; $\mathrm{Com}=$ área de comunicación; $\mathrm{Cog}=$ área cognitiva; $\mathrm{T}=$ puntuación total $\mathrm{PD}=$ puntuación directa; $\mathrm{EE}=$ edad equivalente. 
Tabla 3. Puntuaciones obtenidas con la BLR-E en las evaluaciones realizadas a los 23 y 28 meses de edad cronológica

\begin{tabular}{|c|c|c|c|}
\hline & & $1 E$ & $2 E$ \\
\hline \multirow[t]{3}{*}{$\mathrm{P}$} & PD & 46 & 49 \\
\hline & ED & 17 & 18.15 \\
\hline & $\mathrm{CD}$ & 77.38 & 65.37 \\
\hline \multirow[t]{3}{*}{$\mathrm{C}$} & PD & 65 & 71 \\
\hline & ED & 17 & 19 \\
\hline & $\mathrm{CD}$ & 77.38 & 67.13 \\
\hline \multirow[t]{3}{*}{$\mathrm{L}$} & PD & 22 & 27 \\
\hline & ED & 13 & 15.15 \\
\hline & $\mathrm{CD}$ & 59.18 & 57.12 \\
\hline \multirow[t]{3}{*}{$S$} & PD & 23 & 26 \\
\hline & ED & 14 & 15.15 \\
\hline & $\mathrm{CD}$ & 63.73 & 61.83 \\
\hline \multirow[t]{3}{*}{ G } & PD & 156 & 173 \\
\hline & ED & 15.25 & 17 \\
\hline & $\mathrm{CD}$ & 69.42 & 62.86 \\
\hline
\end{tabular}

Nota: $\mathrm{P}=$ escala postural; $\mathrm{C}=$ escala de coordinación $\mathrm{L}=$ escala de lenguaje; $\mathrm{S}=$ escala de sociabilidad; $\mathrm{G}$ = puntuación global; $\mathrm{PD}=$ puntuación directa; $\mathrm{ED}=$ edad de desarrollo; $\mathrm{CD}=$ cociente de desarrollo.

La Tabla 3 recoge los resultados obtenidos con la escala de desarrollo Brunet-Lézine Revisada (BLR-E) y la Figura 2 presenta las edades equivalentes.

Los resultados clínicos tras la primera aplicación del tratamiento mostraron mejoría del interés por explorar su entorno y se observó aumento del tiempo de atención. Además, el niño empezó a reconocerse a sí mismo como causa de acontecimientos y mejoró su motricidad perceptiva y coordinación óculo-manual (ensartar anillas en un eje, construcción de torres de más bloques, encajables). No se observó evolución significativa en la 
Figura 1. Edades equivalentes obtenidas con el Inventario de desarrollo Battelle

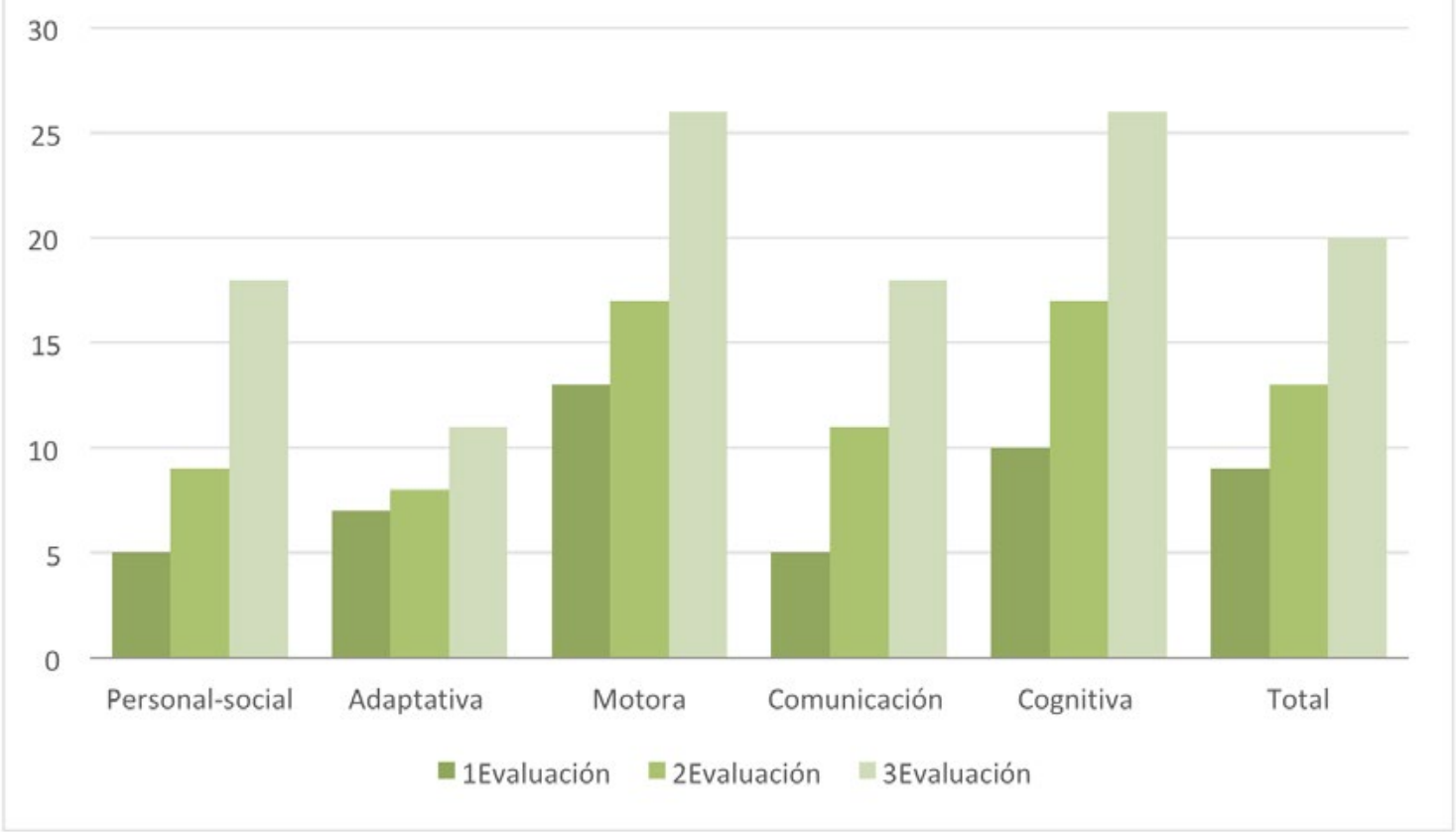

Figura 2. Edad de desarrollo obtenida con la escala Brunet-Lézine Revisada

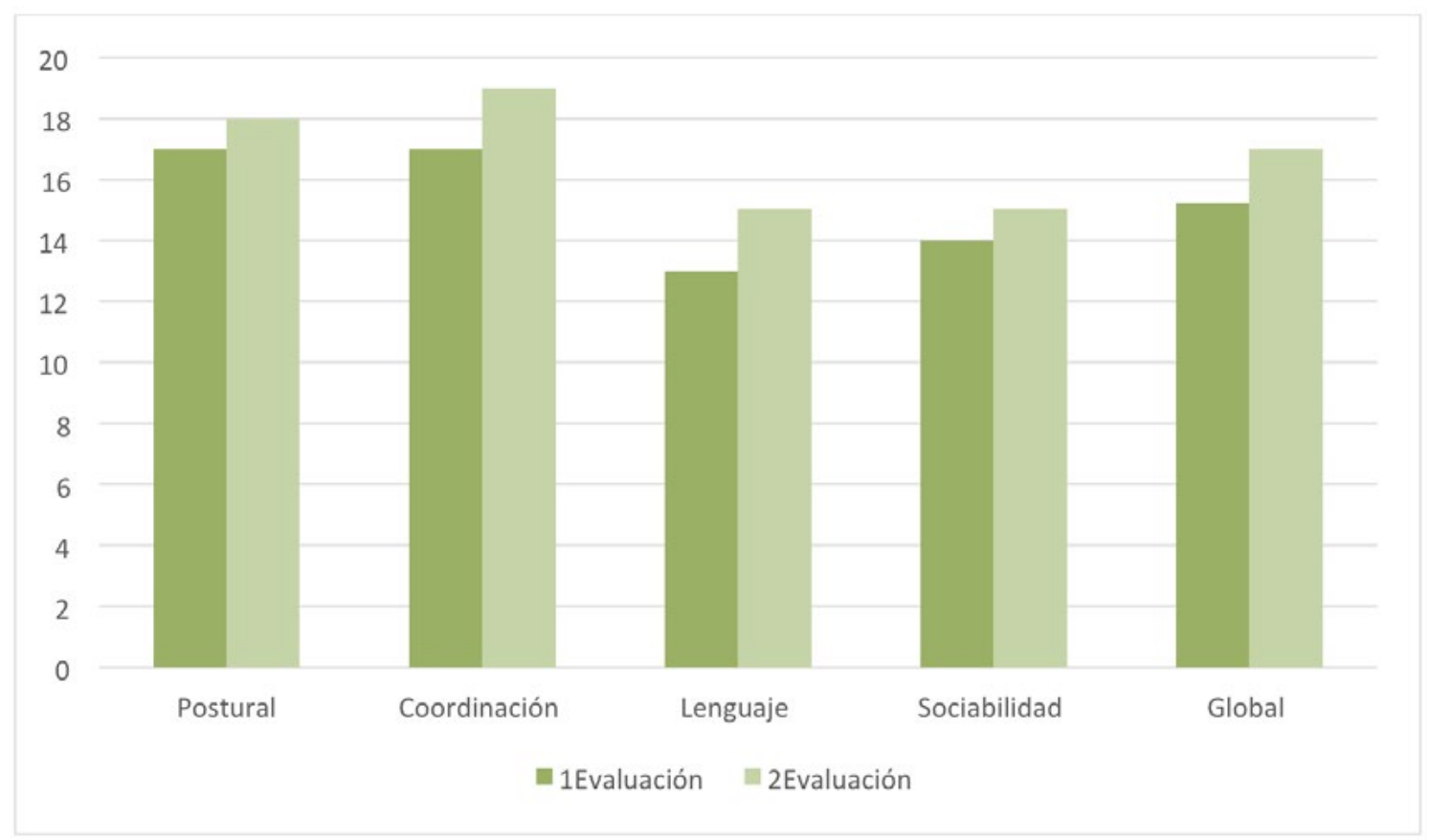

adquisición de conceptos propios de su edad, había iniciado la asociación de colores iguales y el reconocimiento de las partes del cuerpo en sí mismo (boca). A nivel social mejoró el contacto ocular, y su uso como petición o demanda. También se advirtió más atención compartida, juego conjunto e interés por la interacción con otros. Inició el juego simbólico: juego funcional con objetos cotidianos e integración de otros (adultos) en su juego. Se observó mejoras en el seguimiento de normas y en la respuesta a órdenes prohibitivas. Ayudaba a guardar 
sus juguetes. Empezó a buscar recompensas tras sus logros y a repetir acciones que producían una respuesta en el otro (risa, llamadas de atención). Iniciaba la alimentación con semi-sólidos. Los padres comentaban, sin embargo, que habían aparecido nuevas conductas repetitivas: abrir-cerrar puertas y tirar cosas y observar cómo caen. En el área de comunicación-lenguaje cabe señalar que el niño mejoró su intención comunicativa e inició el uso de ademanes para demandar. A nivel comprensivo empezó a seguir órdenes sencillas, con apoyo gestual, conocía el significado de "papá” y "mamá" y asociaba palabras con objetos e ilustraciones. A nivel expresivo, emitió sus primeras palabras con funcionalidad (mama, agua, pompa). A nivel motor ganó estabilidad en la marcha (aunque mantenía base de sustentación aumentada), así como en la subida y bajada de escaleras. Andaba hacia detrás, y se agachaba y levantaba con mayor estabilidad. También mejoró el juego con pelota (lanzar, chutar) y las destrezas de manipulación fina. Ensartaba cuentas grandes, garabateaba con más fuerza, metía-sacaba objetos de un recipiente y abría-cerraba botes. Por otro lado, se observó reducción del nivel de ansiedad durante la exposición a estímulos motores o inestables.

Tras la segunda fase del programa, el menor consiguió diversos avances significativos a nivel clínico. El tiempo de atención sostenida se hacía más prolongado de forma progresiva, se mantenía más tiempo trabajando en mesa durante la propuesta de actividades dirigidas (no así en el trabajo autónomo). Comenzó la discriminación de conceptos análogos y de vocabulario. A nivel comunicativo, el menor mantuvo las palabras conocidas y empezó a generalizarlas, se advirtió mayor jerga e intención comunicativa, pero no se evidenció grandes avances a nivel expresivo. Se valoraba interés por sus iguales, aunque no estrategias de interacción adecuadas ni respeto de turnos. A nivel motor, mostraba importantes avances a nivel de hitos de desarrollo propios de la edad cronológica del niño, aunque se hacía necesario reforzar la coordinación en general, así como las destrezas motoras finas.

\section{Discusión}

Tras la aplicación del programa de intervención específico, se observa diversos avances en las distintas áreas del desarrollo. A pesar de ello, los resultados evidencian la presencia de un retraso generalizado en el desarrollo del menor. Existen dificultades en todas las áreas, siendo las áreas socio-comunicativas y adaptativa las que presentan un mayor desfase en la adquisición de hitos en relación al grupo de edad correspondiente.

No se observa la presencia de cambios numéricos destacables en las distintas evaluaciones; sin embargo, sí resalta la significación clínica de los avances conseguidos. Por otro lado, si se atiende a la puntuación de ganancia o diferencia entre el post-test y el pre-test, se puede valorar cuánto cambio se produce tras el entrenamiento. Según Calero (2004), esta diferencia de puntuaciones en los dos momentos de evaluación es considerada una medida del potencial de aprendizaje.

Todo lo anterior, junto a la influencia que ha demostrado tener la atención temprana en niños con problemas en su desarrollo, apoya la continuidad del tratamiento.

Un aspecto a tener en cuenta para futuras revisiones es el cálculo de las puntuaciones típicas extremas, alternativa que se propone en el manual del inventario Battelle (Newborg et al., 1988) para medir el progreso de un niño cuyos resultados se sitúan en el primer centil (rendimiento por debajo de lo normal para su grupo de edad).

Además de lo anterior, se podría complementar la evaluación con nuevas escalas. Existen pruebas de desarrollo comúnmente utilizadas en atención temprana con baremos de edad superior a los 30 meses (limitación que se ha encontrado en la prueba Brunet-Lézine). Por otro lado, como se ha comentado previamente, entre la sintomatología común en el síndrome de Potocki-Lupski, se ha encontrado muchos rasgos fenotípicos, además de signos relacionados con los trastornos del espectro autista, factor que podría motivar la aplicación de alguna prueba específica para valorar la posibilidad de una presentación comórbida. Bissell et al. (2018), comparan en su estudio el fenotipo conductual del síndrome de Potocki-Lupski y las características propias del TEA, y llegan a la conclusión de que la principal coincidencia resulta en el patrón repetitivo de conductas (tomando un carácter más compulsivo en el caso del síndrome que aquí nos ocupa).

El presente estudio contribuye a la literatura existente sobre el síndrome de Potocki-Lupski, apoya estudios teóricos como los de Potocki et al. (2007), Treadwell-Deering et al. (2010) y Domenico et al. (2019), en los 
que se define la sintomatología común, y añade una perspectiva práctica de cara a la intervención de niños diagnosticados con este síndrome bastante desconocido.

Los autores de este trabajo declaran que no existen conflictos de intereses.

\section{Referencias}

Arnedo, M., Montes, A., Bembibre, J. y Triviño, M. (2015). Neuropsicología infantil. A través de casos clínicos. Editorial médica panamericana.

Asociación Síndrome Potocki-Lupski (2018). https://www.hacesfalta.org/transparencia-ong/detalle/?idOn$\mathrm{g}=18236 \&$ pageIndex $=282$

Bissell, S., Wilde, L., Richards, C., Moss, J. y Oliver, C. (2018). The behavioural phenotype of PotockiLupski syndrome: a cross-syndrome comparison. Journal of Neurodevelopmental Disorders, 10, Artículo 2. https://doi.org/10.1186/s11689-017-9221-X

Bluma, S. y Reinaud, E. (1978). Portage Guide to Early Education [Guía Portage de Educación Preescolar. (E. Espejo trad.)]. Cooperative Educational Service Agency 12. The Portage Project, cop.

Bravo, C., Gamez, F., Perez, R., Aguaron, A. y De Leon-Luis J (2013). Prenatal diagnosis of Potocki-Lupski syndrome in a fetus with hypoplastic left heart and aberrant right subclavian artery. Journal of Perinatology. 3(5), 394-396. https://doi.org/10.1038/jp.2012.77

Brown, A., Phelan, M. C., Patil, S., Crawford, E., Rogers, R. C. y Schwartz, C. (1996). Two patients with duplication of 17p11.2: the reciprocal of the Smith-Magenis syndrome deletion? American Journal of Medical Genetics, 63(2), 373-377. https://doi.org/10.1002/(SICI)1096-8628(19960517)63:2<373::AID-AJMG9>3.0.CO:2-U

Buela-Casal, G., y Sierra, J. C. (2002). Normas para la redacción de casos clínicos. Revista Internacional de Psicología Clínica y de la Salud, 2(3), 525-532.

Cabrera, M., Sahún , M., Martí, M., Martín, M. y Arencibia, H. (2002). Estimulación temprana. Radio ECCA. http://www2.radioecca.org/estudiante/banco-clases/437

Calero, M. D. (2004). Validez de la evaluación del potencial de aprendizaje. Psicothema, 16(2), 217-221. http://www.psicothema.com/pdf/1185.pdf

Candel, I. (2003). Atención temprana. Niños con síndrome de Down y otros problemas del desarrollo. Federación Española del Síndrome de Down.

Candel, I. (2005). Elaboración de un programa de atención temprana. Revista electrónica de investigación psicoeducativa, 3(3), 151-192. https://doi.org/10.25115/ejrep.v3i7.1206

Costas, C. (2009). Evaluación del desarrollo en atención temprana. Revista Interuniversitaria de Formación del Profesorado, 65(2), 21-38.

Domenico, A., Falsaperla, R., Rizzo, R., Ruggieri, M., Verrotti, A. y Pavone, P. (2018). A New Patient with Potocki-Lupski Syndrome: A Literature Review. Journal of Pediatric Genetics, 7(1), 29-34. https://doi. org/10.1055/s-0037-1604479

Fernández, A., Acosta, V. y Revilla, A. (2016). Anestesia en el síndrome Potocki-Lupski/Anesthesia in Potocki-Lupski syndrome (BVS). Revista Española de Anestesiología y Reanimación, 63(9), 548-549. https://doi. org/10.1016/j.redar.2016.07.003

Fuentes-Biggi et al. (2006). Guía de buena práctica para el tratamiento de los trastornos del espectro autista. Revista de neurología, 43(7), 425-438.

Genetic and rare diseases information center, GARD. (2018). https://rarediseases.info.nih .gov/diseases/10145/index Grupo de atención temprana, GAT (2011). Libro Blanco de la Atención Temprana (4⿳亠丷厂 Ed.). Real Patronato sobre Discapacidad.

Josse, D. (1997). Scale psychomotor development of early childhood Brunet-Lezine-Revised [Escala de desarrollo psicomotor de la primera infancia Brunet-Lezine-Revisado. (S. Pereda Marín, Trad.)]. Psymtec. 
Lee, C. G., Park, S. J., Yim, S. Y., y Sohn, Y. B. (2013). Clinical and cytogenetic features of a Potocki-Lupski syndrome with the shortest $0.25 \mathrm{Mb}$ microduplication in $17 \mathrm{p} 11.2$ including RAI1. Brain Development, 35(7), 681-685. https://doi.org/10.1016/j.braindev.2012.09.009

Lee, C. G., Park, S. J., Yun, J. N., Yim, S. Y. y Sohn, Y. B. (2012). Reciprocal deletion and duplication of 17p11.2-11.2: Korean patients with Smith-Magenis syndrome and Potocki-Lupski syndrome. Journal of Korean Medical Science, 27(12), 1586-1590. https://doi.org/10.3346/ikms.2012.27.12.1586

Martin, J., Knight, S.J., Sharp, A.J., Eichler, E.E., Hurst, J. y Kini U. (2008). Potocki-Lupski syndrome mimicking a connective tissue disorder. Clinical Dysmorphology, 17(3), 211-213.

Mazzanati, M. (2011). Declaración de Helsinki, principio y valores bioéticos en juego en la investigación médica con seres humanos. Revista Colombiana de Bioética, 6(1), 125-144.

McCool, C., Spinks, A., Noroski L.M. y Potocki, L. (2017). Potocki-Shaffer syndrome in a child without intellectual disability - The role of PHF21A in cognitive function. American Journal of Medical Genetics Part A, 173(3), 716-720. https://doi.org/10.1002/ajmg.a.37988

Newborg, J., Stock, J. R., Wnek, L., Guildubaldi, J. y Svinicki, J, (1988). Inventario de Desarrollo de Battelle (4 ed.). (Adaptación española: M $^{\mathrm{a}}$ V. de la Cruz y M. González. (2011). TEA.

Pons-Tubío, A. (Coord.). (2009). Proceso seguimiento recién nacido de riesgo: proceso asistencial integrado. Consejería de Salud, Junta de Andalucía.

Potocki, L., Chen, K. S., Park, S. S., Osterholm, D. E., Withers, M. A., Kimonis, V., Summers, A. M., Meschino, W. S., Anyane-Yeboa, K., Kashork, C. D, Saffer, L. G. y Lupski, J. R. (2000). Molecular mechanism for duplication 17p11.2 - the homologous recombination reciprocal of the Smith-Magenis microdeletion. Nature Genetics, 24, 84-87. https://doi.org/10.1038/71743

Potocki, L., Bi, W., Treadwell-Deering, D., Carvalho, C. M., Eifert, A., Friedman, E. M., Glaze, D., Krull, K., Lee, J. A., Lewis, R. A., Mendoza-Londono, R., Robbins-Furman, P., Shaw, C., Shi, X., Weissenberger, G., Withers, M., Yatsenko, S. A., Zackai, E. H., Stankiewicz, P. y Lupski, J. R. (2007). Characterization of Potocki-Lupski syndrome (dup(17)(p11.2p11.2)) and delineation of a dosage-sensitive critical interval that can convey an autism phenotype. American journal of human genetics, 80(4), 633-649. https://doi. org/10.1086/512864

Robles-Bello, M. A. (2016): "Primeros resultados de la evaluación de un programa de Atención Temprana en síndrome de Down”, Revista Española de Discapacidad, 4(1), 53-65. https://doi.org/10.5569/23405104.04.01.03

Robles-Bello, M. A. y Sánchez-Teruel, D. (2006). Evaluación e intervención en atención infantil temprana: hallazgos recientes y casos prácticos. Jaén, ES: Universidad de Jaén.

Robles-Bello, M. A., y Sánchez-Teruel, D. (2013). Atención infantil temprana en España. Papeles del Psicólogo, 34(2), 132-143.

Robles-Bello, M. A. Sánchez-Teruel, D. y Candel, I. (2018). Casos clínicos en centros de desarrollo de atención temprana. Servicios integrados en los ámbitos de Salud, Educación y Social. EOS Psicología.

Robles-Bello, M. A., Sánchez-Teruel, D. y Romero-Prado, A. B. (2014). Perfil de desarrollo evolutivo de un bebé con una crosomopatía infrecuente en nacidos vivos. Apuntes de Psicología, 32(3), 281-288.

Robles-Bello, M. A., Valencia, N., Barba-Colmenero, F. y Sánchez-Teruel, David (2017). Evaluación del perfil cognitivo y de conducta en niños de un centro de atención y desarrollo infantil temprano. Revista Argentina de Clínica Psicológica, 26(3), 313-323.

Sánchez-Teruel, D. (2011). Las enfermedades raras en niños de 0 a 6 años: una aproximación clínica. En M ${ }^{\mathrm{a}}$ A. Robles-Bello y D. Sánchez-Teruel (Coords.). Evaluación e intervención en Atención Infantil Temprana: hallazgos recientes y caso prácticos (pp. 345-382). Servicio de Publicaciones de la Universidad.

Schaeffer, B., Musil, A. y Kollinzas, G. (1980). Total communication: a signed speech program for non-verbal children. Research Press.

Soler-Alfonso, C., Motil, K. J., Turk, C. L., Robbins-Furman, P., Friedman, E. M., Zhang, F., Lupski, J. R., Fraley, J. K. y Potocki, L. (2010). Potocki-Lupski syndrome: a microduplication syndrome associated with oropharyngeal dysphagia and failure to thrive. The Journal of Pediatrics, 158(4), 655-659. https://doi. org/10.1016/j.jpeds.2010.09.062 
Treadwell-Deering, D. E., Powell, M. P. y Potocki, L. (2010). Cognitive and behavioral characterization of the Potocki-Lupski syndrome (duplication 17p11.2). Journal of Developmental Behavioral Pediatrics, 31(2), 137-143. https://doi.org/10.1097/dbp.0b013e3181cda67e

Virués-Ortega, J. y Moreno-Rodríguez, R. (2008). Guidelines for clinical case reports in behavioral clinical Psychology. International Journal of Clinical and Health Psychology, 8(3), 765-777.

Yuan, B., Harel, T., Gu, S., Liu, P., Burglen, L., Chantot-Bastaraud, S., Gelowani, V., Beck, Ch. R., Carvalho, Cl., Cheung, S. W., Coe, A., Malan, V., Munnich, A., Magoulas, P. L., Potocki, L., Lupski, J. R.1. (2015). Nonrecurrent 17p11.2p12 Rearrangement Events that Result in Two Concomitant Genomic Disorders: The PMP22-RAI1 Contiguous Gene Duplication Syndrome. The American Journal of Human Genetics, 97(5), 691-707.https:/doi.org/10.1016/j.ajhg.2015.10.003

Zhang, F., Potocki, L., Sampson, J. B., Liu, P., Sanchez-Valle, A., Robbins-Furman, P. y Lupski, J. R. (2010). Identification of uncommon recurrent Potocki-Lupski syndrome-associated duplications and the distribution of rearrangement types and mechanisms in PTLS. American Journal of Human Genetics, 86(3), 462-470. https://doi.org/10.1016/j.ajhg.2010.02.001

Artículo recibido: 13/10/2019

Artículo aceptado: 10/06/2020 